\title{
Differential Abundances of B-type Supergiants in M33
}

\author{
M.I. Monteverde and A. Herrero \\ Instituto de Astrofísica de Canarias, E-38200 La Laguna, Tenerife, \\ Spain
}

\begin{abstract}
In previous papers we have published preliminary results on the stellar oxygen-abundance gradient in M33 by using B-type supergiants. In the following study, we include one new B-supergiant and derive effective temperatures and gravities for these stars. Determination of atmospheric parameters is based on analyses of silicon ionisation balance.

Detailed non-LTE model atmospheres and non-LTE line formation calculations were used for the abundance determination of $\mathrm{Si}$ and $\mathrm{O}$. Careful differential analyses are used to quantify the differences in metal abundances between M33 stars and MK spectral standards in our Galaxy.

We compare our results with the abundances expected from studies of $\mathrm{H}$ II regions.
\end{abstract}

\section{Introduction}

Radial abundance gradients in spiral galaxies are an important diagnostic in the study of galaxy evolution. As we know from studies in our Galaxy, spectroscopic analysis of B-type stars is an excellent way of determining abundances - see the very recent work on the abundance gradient in the Galaxy by Smartt \& Rolleston (1997), and references therein.

Here, we are dealing with M33, for which there are several abundances studies based on H II surveys. These studies have been summarized by VilaCostas et al. (1992) and Henry \& Howard (1995), and all of them establish a welldetermined abundance gradient for oxygen in M33. It is therefore important to check if the gradient obtained from $\mathrm{H}$ II regions is echoed by stellar spectroscopy.

In Monteverde et al. (1997) we have published first results concerning the stellar oxygen abundance gradient in M33 from B supergiants, and have compared them to results from emission-line spectra of $\mathrm{H}$ II regions. That technique relies on a precise classification of the M33 stars against their galactic counterparts. Now we try to confirm our preliminary results by obtaining stellar parameters and then differential abundances of similar Galactic and M33 supergiants.

\section{Observations and Spectral Classification}

Spectra for 17 objects were obtained in different runs at La Palma by using the 4.2-m William Herschel Telescope and ISIS spectrograph. The spectral resolu- 
tion is about $2 \AA$. Each object was observed in two wavelength regions; the blue one (4000-4600 $\AA$ ) and that of $\mathrm{H} \alpha$, which is a luminosity-sensitive line. Details of the observations and spectra can be found in Monteverde et al. (1996).

Spectral classification of stars in M33 is not as easy as expected, because of a lack of adequate standards of comparison and of variations in metallicity (see Monteverde et al. 1996). A careful spectral classification has indicated that only 9 of the obtained spectra correspond to B-type supergiants and only 6 were suitable for this work.

\section{Method of Analysis}

Our studies are based on non-LTE, plane-parallel geometry in hydrostatic and radiative equilibrium which are not completely realistic physical models for $\mathrm{B}$ supergiants. We will concentrate on a non-LTE differential analysis relative to Galactic standards. Such a differential analysis relies on comparing each star to the appropriate Galactic standard, ensuring that both have similar parameters.

\subsection{Determination of Stellar Parameters}

In our preliminary studies on the oxygen-abundance gradient, the determination of stellar parameters for M33 B stars was made in the following way: the effective temperature was adopted from the spectral type and the microturbulence, $\xi$, was assumed to be that of the Galactic standard counterpart. In the present work we improved the stellar parameter determination by using ionization balance due to Si III / Si IV and Si II / Si III which are available for use as indicators of $T_{\text {eff }}$ in B-type supergiants. Silicon lines were also used to derived $\xi$ values.

The atmospheric parameters $T_{\text {eff }}, \log g,[\mathrm{He}],[\mathrm{Si}]$ and $\xi$ are not independent and a degree of iteration is required. The analysis of the hydrogen and silicon spectra allows a consistent determination of the effective temperature, the gravity, the microturbulence and the unknown silicon abundance. We have assumed a solar helium abundance for all our supergiants. Preliminary values of $\log g$ and $T_{\text {eff }}$ were first determined. As Balmer lines are almost unaffected by $\xi, \mathrm{H} \delta$ and $\mathrm{H} \gamma$ have been used as indicators of $\log g$. For each grid value of $T_{\text {eff }}$ we

Table 1. Non-LTE Atmospheric parameters for the programme stars, derived from the silicon ionisation balance.

M33 Blue Supergiants

\begin{tabular}{lcccccc}
\multicolumn{1}{c}{ Star } & Id. & Spec. Type & $T_{\text {eff }}$ & $\log g$ & $\xi$ & Ion. balance \\
\hline 0785 & $110 \mathrm{~A}$ & B1 Ia $^{+}$ & 19000 & 2.20 & 19 & Si III/Si IV \\
ob21-108 & B38 & B1.5 Ia $^{+}$ & 20700 & 2.37 & 20 & Si III/Si IV \\
1054 & & B2 Ib & 21000 & 2.55 & 9 & Si II/Si III \\
0515 & B133 & B2.5 Ia & 21300 & 2.53 & 15 & Si III/Si IV \\
\hline \hline
\end{tabular}

Standard Galactic Comparison Stars

\begin{tabular}{llcccccc}
\hline \multicolumn{1}{c}{ Star } & Spec. Type & $T_{\text {eff }}$ & $\log g$ & $\xi$ & Ion. balance & $12+\log (\mathrm{Si} / \mathrm{H})$ & $12+\log (\mathrm{O} / \mathrm{H})$ \\
\hline HD13854 & B1Iab & 21700 & 2.52 & 14 & Si III/Si IV & $7.55 \pm 0.20$ & $8.90 \pm 0.13$ \\
HD14956 & B1.5Ia & 19300 & 2.28 & 15 & Si III/Si IV & $7.87 \pm 0.15$ & $8.83 \pm 0.15$ \\
HD206165 & B2Ib & 20000 & 2.45 & 15 & Si II/Si III & $7.25 \pm 0.10$ & $8.45 \pm 0.10$ \\
HD198478 & B2.5Ia & 18500 & 2.20 & 8 & Si II/Si III & $7.51 \pm 0.05$ & $8.35 \pm 0.20$ \\
\hline \hline
\end{tabular}


have determined a unique $\log g$, for which the observed and the theoretical line profiles agreed best. For each of those $\left(\log g, T_{\text {eff }}\right)$-pairs we calculated silicon line profiles at different $\xi$. A, solar silicon abundance is initially assumed. From the diagram of theoretical equivalent width ratios for each pair of silicon lines we determine $\log g, T_{\text {eff }}$ and $\xi$ by comparing with the observed equivalent width.

Then we considered fixed the preliminary values of $\log g$ and $T_{\text {eff }}$ and the microturbulence and silicon abundance are freely varied in order to ensure that the derived abundances from individual lines are not a function of strength. Si III lines, which come from a single multiplet, were chosen to create the diagram of silicon abundance against observed equivalent width. The new value of microturbulence is that one which produces a zero slope in the diagram and the corresponding $\mathrm{Si}$ abundance comes from the value indicated by that zero slope.

The so defined silicon abundance served as basis for a new $\left(\log g, T_{\text {eff }}, \xi\right)$ determination. These steps are repeated until the change of $T_{\text {eff }}, \log g, \xi$ and silicon abundance was within the error in the equivalent width measurements.

\subsection{Metal Abundances}

We generated non-LTE model atmospheres for each star with the atmospheric parameters listed in Table 1 . The subsequent non-LTE line-formation calculations were performed using the programs DETAIL and SURFACE. As regards the oxygen and silicon atomic models, we used the model for O II set up by Becker \& Butler (1988) and for Si II , Si III and Si IV by also Becker \& Butler (1990). Non-LTE curves of growth were constructed for the lines of interest for each element, using a range of abundances. The results of the abundance analysis for the galactic standards are given in Table 1 . Table 2 gives the differential abundance for the M33 stars.

\section{Oxygen and Silicon Abundance Gradients in M33}

The silicon and oxygen non-LTE differential abundances for the M33 B-type supergiants compared to galactic standards with similar atmospheric parameters are listed in Table 2, along with their projected galactocentric distance in arcminutes, $\rho$.

A comparison of our $\mathrm{O}$ II results with those from $\mathrm{H}$ II studies is made in Fig. 1 (left). The oxygen differential abundances for M33 stars obtained here are represented by asterisks. The line represents the fit of Henry \& Howard (1995) to $\mathrm{H}$ II regions data from different authors, and diamonds give individual

Table 2. Silicon and oxygen non-LTE differential abundances for the M33 B-supergiants compared to galactic standards.

\begin{tabular}{llccc}
\hline M33 Star & Standard & $\rho$ & $\log (\mathrm{Si} / \mathrm{H})-\log (\mathrm{Si} / \mathrm{H})_{\odot}$ & $\log (\mathrm{O} / \mathrm{H})-\log (\mathrm{O} / \mathrm{H})_{\odot}$ \\
\hline 1054 & HD206165 & $1^{\prime} .0$ & $+0.25 \pm 0.14$ & $+0.27 \pm 0.18$ \\
B133 & HD13854 & $9^{\prime} .4$ & $-0.55 \pm 0.26$ & $-0.80 \pm 0.24$ \\
ob21-108 & HD14956 & $11^{\prime} .7$ & $-0.67 \pm 0.20$ & $-0.40 \pm 0.23$ \\
110A & HD14956 & $17^{\prime} .1$ & $-0.59 \pm 0.20$ & $-0.34 \pm 0.33$ \\
\hline \hline
\end{tabular}



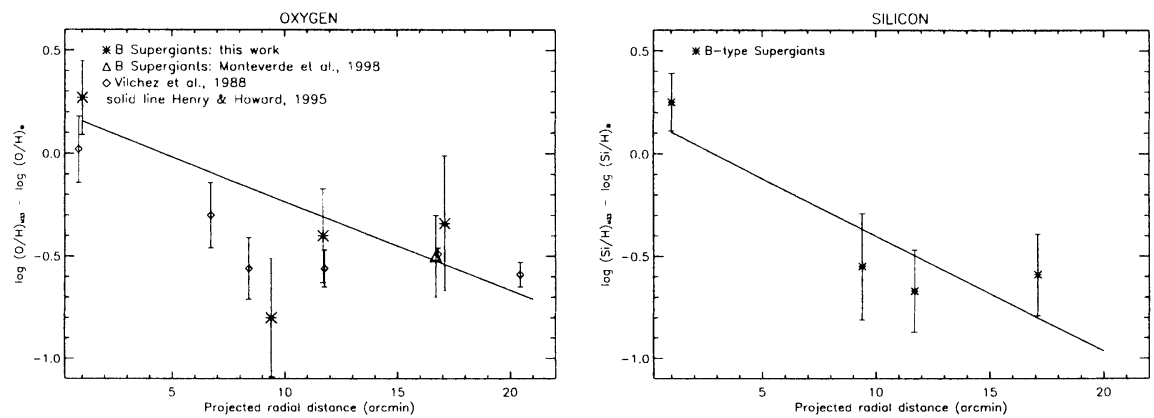

Figure 1. (left) The oxygen differential abundance as a function of galactocentric distance for M33 stars. See text for symbols explanation. (right) The silicon differential abundance for M33 stars.

points by Vilchez et al. (1988). The triangle represents one more point from Monteverde (1998).

Figure 1 (right) shows the differential silicon abundances for the programme stars (asterisks) and the solid line represents the silicon gradient from the fit to the points.

\section{Conclusions}

We have confirmed here, with a more reliable stellar parameters determination, the first results by Monteverde et al. (1997) and Monteverde (1998) concerning the oxygen abundance gradient in M33 from B supergiants. The main results are:

a) We find consistency for old and new oxygen abundances in B supergiants (except for B133).

b) We confirm that our $\mathrm{O}$ gradient is in good agreement with $\mathrm{H}$ II region results.

c) A comparison of differential silicon and oxygen abundances within each star indicates they are well correlated.

d) The $\mathrm{O}$ and $\mathrm{Si}$ gradients obtained are compatible with each other. We obtain $-0.17 \pm 0.36 \mathrm{dex} / \mathrm{kpc}$ for $\mathrm{O}$ and $-0.27 \pm 0.25 \mathrm{dex} / \mathrm{kpc}$ for $\mathrm{Si}$.

We are planning to complete this work with the analysis of two more M33 B-type supergiant along with a magnesium abundance determination. Also new, better observations with $10 \mathrm{~m}$-class telescopes will help to reduce the large errors, dominated by the equivalent widths measurements.

\section{References}

Becker, S.R., Butler, K. 1988, A\&AS, 201, 232

Becker, S.R., Butler, K. 1990, A\&AS, 235, 326

Henry, R.B.C., Howard, J.W. 1995, ApJ, 438, 170

Monteverde, M.I., Herrero, A., Lennon, D.J., Kudritzki, R.P. 1996, A\&A, 312, 24 
Monteverde, M.I., Herrero, A., Lennon, D.J., Kudritzki, R.P. 1997, ApJ, 474, L107

Monteverde, M.I. 1998, in: Boulder-Munich II: Properties of Hot, Luminous Stars, (ed.) I.D. Howarth, ASP Conf. Ser., 131, p. 188

Smartt, S.J., Rolleston, W.R.J. 1997, ApJ, 481, L47

Vila-Costas, M.B., Edmunds, M.G. 1992, MNRAS, 259, 121

Vilchez, J.M., Pagel, B.E.J., Díaz, A.I., Terlevich, E., Edmunds, M.G. 1988, MNRAS, 235, 633 